International Journal of Wireless \& Mobile Networks (IJWMN) Vol. 4, No. 6, December 2012

\title{
CoORdinating Disaster RELIEF OPERATIONS USINg SMART PHONe / PDA BASEd PEer-To- PEer Communication
}

\author{
Debanjan Das Deb ${ }^{1}$, Sagar Bose $^{2}$ and Somprakash Bandyopadhyay ${ }^{3}$ \\ ${ }^{1}$ Department of Computer Science \& Engineering, \\ B. P. Poddar Institute of Management \& Technology, India \\ debanjandasdeb@gmail . com \\ ${ }^{2}$ PervCom Consulting Pvt. Ltd., India \\ sagar@pervcomconsulting.com \\ ${ }^{3}$ Indian Institute of Management Calcutta, India \\ somprakasheiimcal.ac. in
}

\begin{abstract}
During any post-disaster period, the availability of the Internet is ruled out in most cases, mobile phones are only partially usable in some selected regions. Candidate devices for maintaining minimal services are mostly expensive satellite phones or specialized point-to-point radio communication systems. As communication systems become crippled, so do the management of the relief operations. One of the common problems during disasters is that the rescue and relief operations are not well-coordinated. For this reason, there is a need for a system that will help in the efficient distribution of rescue and relief to disaster-affected areas. The objective of this paper is to propose a smart-phonel PDA based disaster management system based on peer to peer communication only and supporting disconnected operation.
\end{abstract}

\section{KEYWORDS}

Peer-to-Peer Communication, Opportunistic Network, Delay-tolerant network

\section{INTRODUCTION}

Any large-scale disasters like flood and cyclone have severe impact on communication infrastructure. Services that are relied on for everyday communications (e.g., cell phone / internet connectivity) immediately become non-functional in emergency situations due to the failure of the supporting infrastructure through both system damage and system overuse [1]. In contrast to the vulnerable fixed network infrastructure, it is very likely that battery-powered wireless personal mobile communication devices (PDA, cell-phones) will survive in disasters. Additionally, even more mobile devices will be brought to the scene by relief workers. Currently, those devices are having powerful processors and high storage capacity with GPS and multi radio interfaces (Cellular, Wi-Fi, Blue-tooth). Such devices are, therefore, promising candidates to contribute in forming peer-to-peer wireless network structure to support disaster communication.

However, end-to-end connectivity can never be assumed in this kind of scenario and long disconnections are the rule. Thus, in this context, the devices (PDA, cell-phones) spread across the environment by relief workers form peer-to-peer network, albeit disconnected. In this type of networks, the mobility of devices is an opportunity for communication rather than a challenge. Mobile nodes communicate with each other in peer-to-peer mode. 
The objective of this paper is to develop a dynamic, virtual star topology with static central control station as root node and static shelter points as end-nodes (figure 1). The connectivity between root node and each of the end-nodes is achieved using mobile volunteers opportunistically as message ferry. At the same time, the mobile volunteers also exchange information among one another in a peer to peer mode, thus integrating the field information intelligently and autonomously using auto-configurable mobile-phone-based peer-to-peer communication. Basically mobile volunteers act like end devices. The major focus of this study is to come up with a framework for post-disaster resource requirement analysis, resource allocation \& distribution, which involves on-going determination of what resources are needed, what resources are present, what resources need to be acquired and how long will it take for them to arrive. Using peer-to-peer communication, eventually the entire network will appear as virtually connected, delay-tolerant network with every node knows the approximate information about other nodes, including resource need. Thus, the system enables coordinated relief operation, where no shelter will be having excess resource, nor it will be under-resourced.

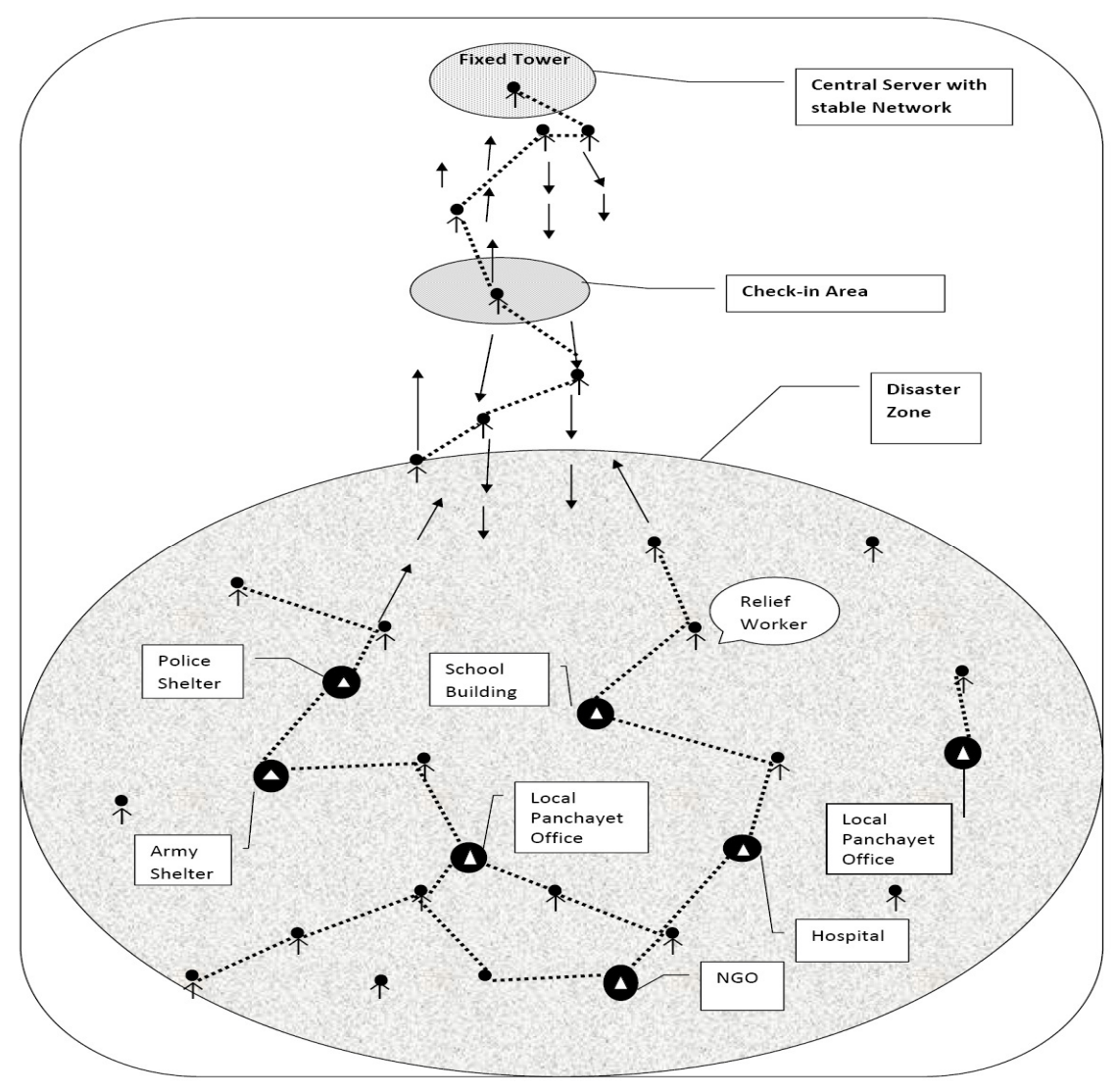

Figure 1. Framework for post-disaster communication between Central Server, Shelter and Volunteers

\section{RELATED WORK}

In disasters, it is necessary to have established procedures for obtaining additional resources when they are needed. However, indiscriminate requests for resources can be detrimental. Many disasters are complicated by the over-response of resources, and this can greatly complicate the already difficult problems of coordination and communication. Procedures for pinpointing the specific types and numbers of resources needed are helpful in making the disaster response 
International Journal of Wireless \& Mobile Networks (IJWMN) Vol. 4, No. 6, December 2012

more manageable. Disasters with multiple impact sites and large, complex disasters often call for an emergency operations center (EOC) (Erik, 1989) [4]. The EOC or Central Control Station is usually established away from the disaster scene, often near governmental offices in contrast to the command post, which is concerned with activities at the scene, the EOC establishes priorities for the distribution of resources among the various sites, and handles off-incident concerns. This is basically a centralized system for controlling disaster relief management. However, a major concern is the connectivity between EOC and the shelter points in the field.

"VSAT technology", which is particularly useful when terrestrial infrastructure has been destroyed, provides a powerful tool to mitigate damage incurred by disasters (Gorp, 2008) [5]. The deployment of VSAT is typically seen as the last option for obtaining access to larger communications network and the Internet. It is often necessary for organizations operating in remote areas. However, VSAT Technology is expensive and its deployment is complex in nature. Technical support is critical for VSAT deployment after disasters, because in the disaster area the people are much more focused on helping people than messing with technology. VSAT is often deployed in the context of establishing new field offices; and thus is primarily deployed for development purposes, rather than for emergency response, or direct post-disaster relief.

Multiple-Criteria Decision Technique, together with a coordination strategy and a communication strategy, have been deployed in order to assure that the decision making process has the appropriate information upon which to perform the resource distribution (Silvia, 2003) [6]. The coordination strategy allows distributing resources to the victims that need the most urgent rescue. The communication strategy emphasizes information flow concerning disaster victims. The role of the moving agents is to gather information about victims and the role of the fixed agents is to pass on this information to the fixed stations. Here, some communication infrastructure has been assumed. However, if there is no communication at all between agents, then results show that moving agent get lost in the rescue scenario and cannot find victims.

Cluster-based Communications System focuses on the vital need for providing communications facility to the victims, immediately after the disaster and prior to the arrival of rescue teams (Sonia, 2009) [7]. The proposed novel approach in emergency communications enables survivors to communicate among themselves and help each other. Here, the idea of a self organizing ad hoc network is put forward, which makes use of available network resources formally occupied by the destroyed and / or damaged telecommunication infrastructure. In postdisaster scenario, mobile nodes establish a self-organizing / ad hoc network which provides critical level of communications among disaster victims needed at that time and consequently tries to merge with some surviving telecommunications infrastructure and / or network deployed by rescue teams. But, depending on the nature of disaster, a connected ad hoc network may never be formed, and thus communication among victims cannot be established.

Prototypical Crisis Information Management System (Iannella, 2007) [8] is a conceptual framework to support two challenges: incident notification and resource messaging. The mantra for Crisis Information Management Systems is to "deliver the right information to the right people in the right format in the right place at the right time". These five variables, coupled with the stress of a major disaster, make coordinated information management one of the greatest challenges for the disaster coordination sector. However, this system assumes that a strong ICT infrastructure is in place in order to enable cooperation between a large number of organizations and integration with these organizations. A similar collaborative mechanism has been proposed by Marrella (2011) [9], where the communication is executed on top of mobile networks. However, the collaboration strictly depends on the possibility that operators and their devices can communicate with each other using some communication backbone. 
Starting from collected user requirements and their generalization, WORKPAD architecture (de Leoni, 2007) [10] is based on a 2-levels peer-to-peer (P2P) paradigm: the first P2P level is for the front-end and the latter level is for the back-end. The need of such two P2P levels arises from the analysis of user requirements, as there exist back-end central halls where the chiefs of involved organizations are located, as well as several front-end teams which are sent to the affected area. This architecture is based on a stable centrally connected network where all the information percolates and centrally managed. If the entire connection disrupted then the efficiency level will decrease drastically.

\section{SYSTEM ARCHITECTURE}

\subsection{System Description}

During disaster, victims of a village / locality normally take shelters in groups in some nearby safe areas (for example, some school buildings, Army-Police camp and Panchayet Offices in some highland areas, etc.). Therefore, coordination of post-disaster relief operations and distribution of resources are shelter-centric (figure 2) through the relief-workers working at those shelters (figure 3). These relief workers are exchanging their presence, field information and shelters requirements by using their smart phones in an opportunistic network. These shelters are operated by a Shelter Coordinator who will periodically coordinate with nearest relief workers autonomously for analysing field need and informing shelter need through smart phone. Another role of shelter coordinator is to control the navigation pattern of nearby relief workers with an objective to spread the information to other shelters and eventually to Central server. Thus the information percolates from lower layer to upper layer through relief workers.

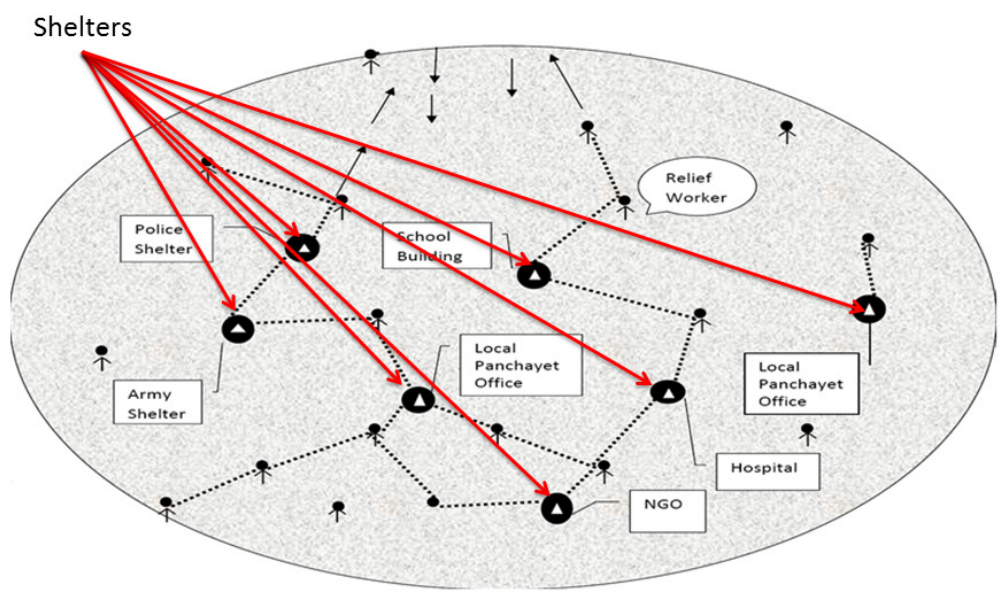

Figure 2. Shelters in the affected zone 


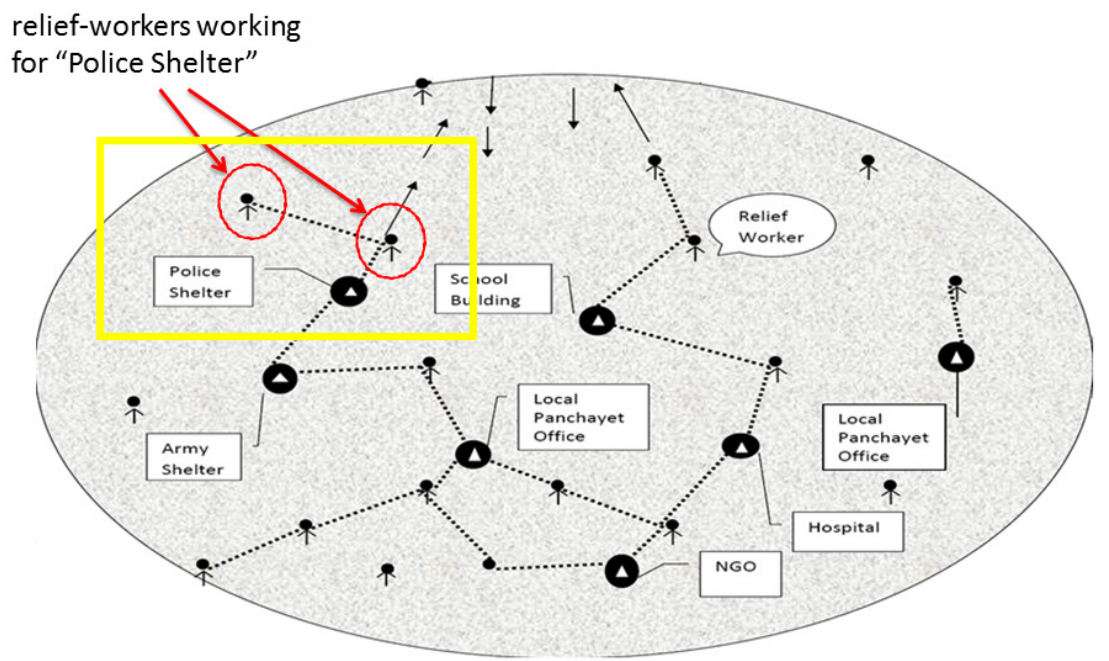

Figure 3. Relief workers working at shelters

The shelter coordinator redirects relief workers by evaluating the field requirement with a primary intention to send at least a few workers towards central server. Once relief workers are get connected with another relief worker, the information exchanges and the modified information percolated in multi hop. Central server also sends few relief workers with relief materials towards disaster area through warehouse. This central server is established in an area where it gets proper communication network. It receives information from relief workers and sends its directions to the relief worker who is nearer to central server and then that relief worker exchange that information / resource (relief materials) to its nearer worker and by this way the total information spreads out all over the affected area (figure 4).

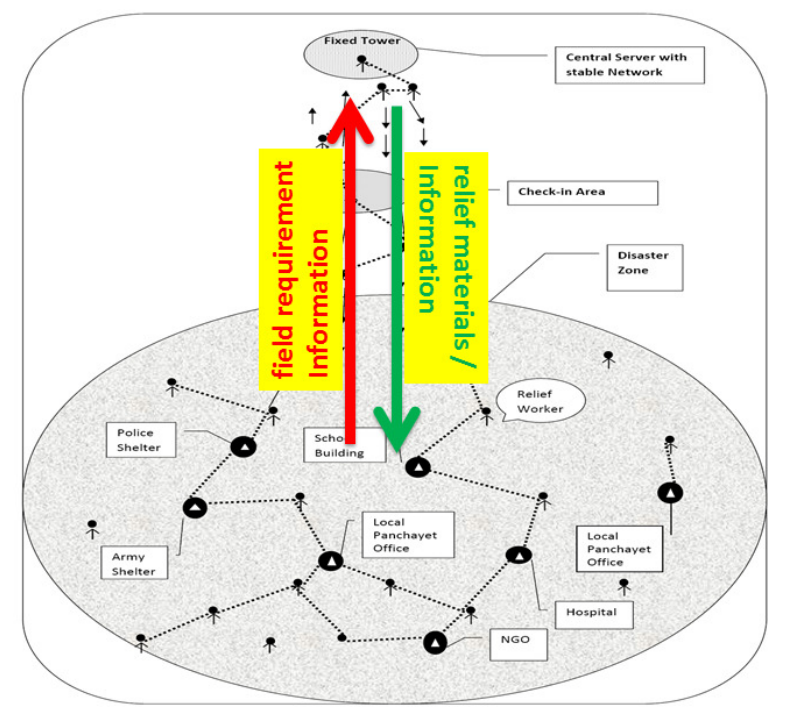

Figure 4. System coordination by Central Station

\subsection{Formation of Smart phone based Opportunistic Network}

In the decentralized Disaster Management scenario, relief workers are exchanging their presence, field information and shelters requirements by using their smart phones in an opportunistic network. Opportunistic network as one type of challenged networks where 
International Journal of Wireless \& Mobile Networks (IJWMN) Vol. 4, No. 6, December 2012

network contacts are intermittent or where link performance is highly variable or extreme. In such a network, there does not exist a complete path from source to destination for most of the time. In addition, the path can be highly unstable and may change (make or break) quickly. Therefore, in order to make communication possible in an opportunistic network, the intermediate nodes may take custody of data during the blackout and forward it when the connectivity resumes. Thus, in this context, Opportunistic Network framework [2,3] provides potential platform for information communication. In opportunistic networks, the devices (PDA, cell-phones) spread across the environment by relief workers and form the network. In this type of networks, the mobility of devices is an opportunity for communication rather than a challenge. Mobile nodes communicate with each other even if an end-to-end route connecting them never exists. Any node can opportunistically be used as the next hop, if it is likely to bring the message closer to the destination(s).

Currently, those devices or Smart Phones are having powerful processors and high storage capacity with GPS and multi radio interfaces (Cellular, Wi-Fi, Blue-tooth). These Smart Phones are not only useful for making telephone calls, but also adds features that might be found in a personal digital assistant or a computer. Smartphone also offers the ability to send and receive messages on peer to peer basis using GPS or Wi-Fi, Blue-tooth technologies and edit Office documents. Such devices are, therefore, promising candidates to contribute in forming ad-hoc wireless network structure to support disaster communication.

In a seminal paper in Physical Review Letters, Vicsek et al. $[13,14]$ propose a simple model of $n$ autonomous agents moving in the plane with the same speed but with different headings. Each agent's heading is updated using a local rule based on the average of its own heading plus the headings of its "neighbors." In their paper, Vicsek et al. demonstrated that the nearest neighbor rule can cause all agents to eventually move in the same direction despite the absence of centralized coordination and despite the fact that each agent's set of nearest neighbors change with time as the system evolves. Other studies also indicate that multi-agent systems that interact through nearest-neighbor rules can synchronize their states regardless of the size of communication delays [15]. We have applied this concept in our system and the performance evaluation results indicate the effectiveness of our approach.

\subsection{Decentralized Information Assimilation \& Coordination}

In this scenario the information generated on peer to peer basis between relief workers and static shelter points by using opportunistic network. This information percolates from lower layer to upper layer through shelter belonging to same cluster (figure 5).

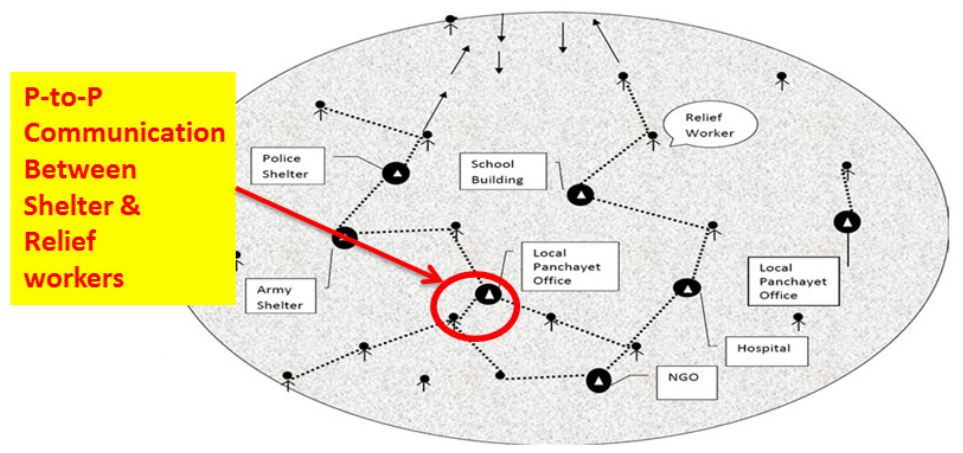

Figure 5. Peer-to-peer communication (all dotted lines) between shelter and relief workers 
The Shelter leaders redirect relief workers by evaluating the field requirement with a primary intention to send at least a few workers towards central server. Once relief workers are get connected with another relief worker, the information exchanges and the modified information percolated in multi hop (figure 6).

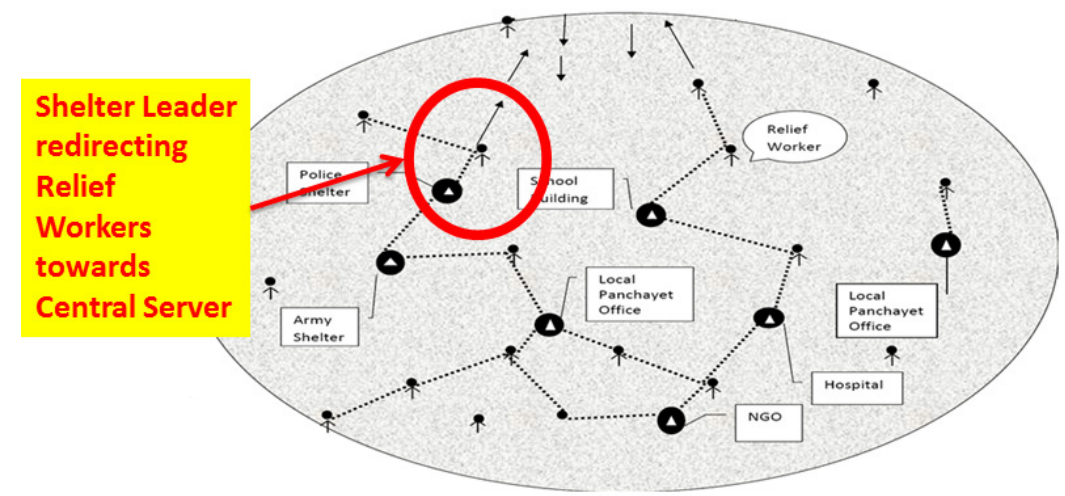

Figure 6. Shelter Leader / coordinator redirecting relief workers towards Central Server

Central server also sends few relief workers with relief materials towards disaster area through check-in-area. This central server is established in an area where it gets proper communication network. It receives information from relief workers and sends its directions to the relief worker who is nearer to central server and then that relief worker exchange that info to his nearer worker and by this way the total information spread out to entire area (figure 7).

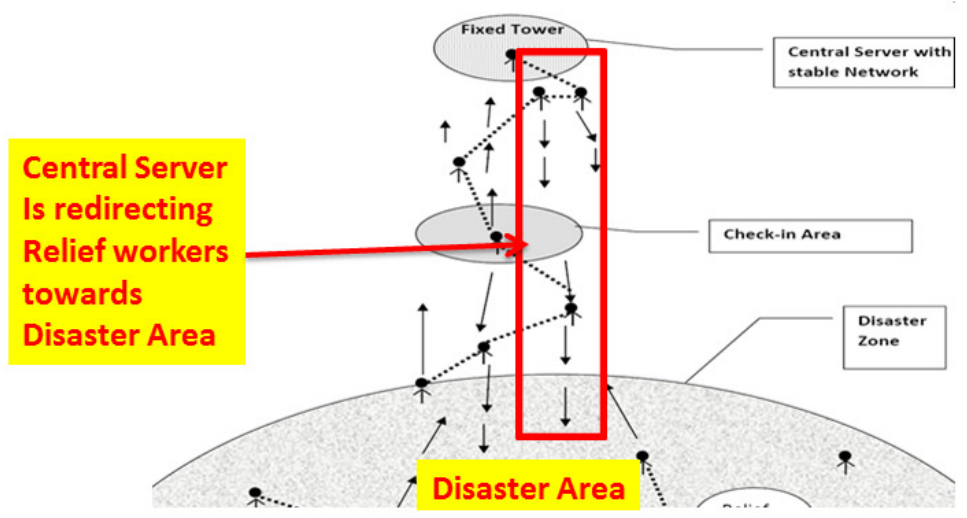

Figure 7. Central Server is redirecting relief workers towards disaster area

The static shelter coordinator sends information by using Smart Phone to nearer relief worker. This information contains sender ID, location and sending time which will help to review the information time and identification status. Relief worker also has the option to forward that received information from shelter coordinator to another relief worker by updating its part of information like:

- Id

- Location of work

- Status of work (whether pending or will take time) 
International Journal of Wireless \& Mobile Networks (IJWMN) Vol. 4, No. 6, December 2012

- Navigation status (if the shelter coordinator has instructed to navigate to central server or any other shelter)

- Infrastructure information

- Information related to difficulties faced

- Necessary resource requirement

Shelter coordinator also spread out information of:

- Infrastructure towards his shelter with location specification.

- Victim's status like

- how many of them are still alive,

○ how many are required hospitalization

- Details of resource requirement and present resource status of the shelter.

- Navigation request: At first it will judge shelter's resource requirement (not only its shelter but also other neighbouring shelters) then depending on the situation it will redirect relief workers towards shelters or towards central server.

Central server also sends its instruction or information in multi hop through relief workers who are approaching towards disaster zone by spreading information through smart phone on Peer to Peer (P2P) basis. It also specifies the sending time, ID and location in its message. Relief workers are not able to modify that information. They can only exchange information by forwarding that message coming from Central server. In this case, relief workers have the option to modify the forwarding time, location and its Id only. Depending on that, any receiver of that message can easily filter the information coming from central server on time and location basis. The main contents of message from central server are

- Resource sending status like quantity of resources have already sent for specific shelter

- Request towards any other shelter to navigate its resources towards that specific shelter

- Request for emergency services like the necessary initiatives should be taken by Police, Army, Doctors, NGO professionals, and Fire Bridget for any specific location

\section{- Instruction for shelter coordinator}

This is a random process by which the total information percolates to entire disaster region. It will create a standard data base on which a specific software model can be established for retrieving required information by filtering this. Relief workers will get an option to save the received messages on its phone and by saving all these information, there will create a broad data base for them where the information will automatically be updated by saving it and repeated information will automatically get erased. 
International Journal of Wireless \& Mobile Networks (IJWMN) Vol. 4, No. 6, December 2012

Central server will get the entire disaster zone related information from different relief workers periodically and will create a broad data base depending on that information in its system. This data base will be circulated to outside world for their knowledge and for asking necessary support from outsiders. The formation of check in area is an activity of central server which will work as a central wire house for keeping relief resources systematically and send them periodically towards disaster zone when these are required. This check in area will work as a manager for the distribution of resources properly. It is an area where initially all relief workers will accumulate and then will spread out to whole disaster zone. To reduce the pressure on Central Server this Check-in-Area will work as a coordinator between central server and main disaster zone. The segregation of work will standardize the pattern of distribution and communication in a decentralized disaster environment. However, there is no fixed network established in the disaster area.

\section{Simulation}

\subsection{Simulation set up}

We are interested in studying a post disaster scenario in which the resources and aid can effectively be distributed to the shelters being formed amidst the disaster affected area. The environment consists of few entities of our concern that are essential in studying the scenario. They are the volunteers or relief workers which co-ordinately achieve some tasks and form the only medium for dispersion of information throughout the affected area. There are fixed locations or relief camps at certain points known as shelters where victims take shelter and it is their needs that the volunteers ensure to fulfil. There is a fixed location outside the affected site known as the Check-in-Area (CA) from which coordinated and centralized relief operations are being monitored. There is no direct network connectivity among CA and shelter points. Next we have another static entity known as the fixed Central Server which acts as a resource pool and a medium to connect to the outside world. CA and Central Server also have no network connectivity.

Since there are few categories among them which performs the same tasks and have the same attributes, the best way to represent them in a similar environment is to incorporate them as agents and a very powerful toolkit that comes handy in this perspective is REPAST Symphony. Recursive Porous Agent Simulation Toolkit (REPAST) is a free and open source agent-based modelling toolkit that offers users with a rich variety of features $[11,12,13,14]$. This simulated environment is written in JAVA.

\subsection{Simulation parameters}

- The proposed scheme is evaluated on a simulated environment by varying key parameters related to the model to study and estimate the efficiency and uniformity of resource distribution against time-ticks.

- In the simulation, the environment to be studied is assumed to be an area of $10 \mathrm{k} . \mathrm{m} . \mathrm{x}$ 10 k.m. in which mobile volunteers are initially distributed randomly. However, the affected site is considered to be a rectangular area of size $10 \mathrm{k} . \mathrm{m}$. x 4 k.m. in which all the shelters are present.

- For all cases we have kept the shelter count constant and it equals 10. All the simulated actions are associated with time-ticks.

- In the Repast platform, "ticks" are considered as a unit of time; we have assumed 1 tick equals 1 minute and hence the speed of movement of individual volunteers is considered to be 100 meters per minute. 
International Journal of Wireless \& Mobile Networks (IJWMN) Vol. 4, No. 6, December 2012

- In this environment, relief resources like food, medicine, clothes, etc. is considered as an aggregated integer quantity, called resource-count.

- The positive resource-count indicates that resources are present in excess.

- The negative resource-count indicates that there is a demand of resources at the shelter and no resource is present.

- If resource-count equals zero, it means that neither there is a demand nor there is any surplus of resource at that shelter.

\subsection{Simulation environment overview}

- The volunteers move randomly at the beginning.

- As indicated, each shelter has either positive or negative resource. Each shelter autonomously decrements the resource-count periodically (self-decrement of resourcecount), indicating that victims present at the shelters are consuming resources and the net resource- count at each shelter decreases randomly with time.

- When a volunteer visits a shelter whose resource-count is negative (i.e. when there is a demand), then it goes directly to the CS, to give that information and to get the needed resource physically. In this way, the CS gets the information of resource-need and resource distributed to all the shelters. The shelter ensures that no other volunteer visits with the same piece of information to the CS.

- When a volunteer goes to the CS with negative resource information, then at separate times of the simulation, two cases might arise :

I. At the initial phase of the simulation, the CS may start giving out resources from itself in a little excess quantity than required until there are enough shelters being discovered with excess resources.

II. After there are plentiful of references to the shelters being discovered, only then the CS assigns tasks to the respective volunteers involved to transfer resources from a nearby shelter having positive resources to the shelter with negative resources.

- It is assumed that each volunteer is carrying PDA / smartphone with Wi-Fi / Bluetooth interface for peer-to-peer communication. It is also assumed that the cellular communication infrastructure is not functioning and volunteers are exchanging information only in peer-to-peer mode.

- At first volunteers have no knowledge about other volunteers and location of shelters. They move randomly in the disaster area to discover shelters and other volunteers. When a volunteer roaming randomly comes near a shelter or the shelter is within the vision of the volunteer, then the volunteer checks its own queue (the queue storing shelter IDs).

- If that particular shelter is present in the queue, it signifies that this volunteer had recently visited that shelter. So, no need to again visit that shelter until recently but exchange information with that shelter. And again the volunteer starts roaming randomly. 
International Journal of Wireless \& Mobile Networks (IJWMN) Vol. 4, No. 6, December 2012

- If there is no occurrence of the shelter in its list then the volunteer approaches the shelter and waits there until the tag of the shelter (previously described) becomes false.

- The same procedure is performed while another roaming volunteer comes under the vision of this volunteer. Both exchange information. In this case, if the occurrence of meeting with the other volunteer is present in this volunteer (queue) then, it will not exchange information because, it is probable that more or less the information might be redundant.

- Before going further it is needed to describe the data structures and the type of data that a volunteer, shelter and Central Station keeps with them. They are discussed below :

\section{I) Data kept by a volunteer:}

- Name (or ID) and location of the shelters known to it.

- Name (or ID) of other volunteers present and known only to this volunteer.

\section{II) Data kept by a shelter:}

- Name (or ID) and location of other shelters present in the affected area known to it.

- Name (or ID) of other volunteers present and known only to this shelter.

- It keeps all the timestamps history of when (at what time-tick) a specific volunteer had visited this shelter (required to track a volunteer).

\section{III) Data kept by the central station:}

- Similar to shelter and volunteers it also keeps a list of shelters and volunteers.

- Name (or ID) and location of all shelters present in the affected area known to it. Initially, it is empty and is updated by the volunteers.

- Name (or ID) volunteers present in the affected area. Initially, it is empty and is updated by the volunteers.

- A shelter's resource status list that stores the status of resources at the shelters known to the CS through interaction with the volunteers.

- While roaming, the volunteer also checks the status of the shelter (a Boolean flag); two cases might occur at this moment:

- If the flag is found true (it means some other volunteer is working for this shelter to fetch and transfer resources ), then it waits for a certain number of time-ticks describing the fact that it is both waiting for the flag to get false and 
International Journal of Wireless \& Mobile Networks (IJWMN) Vol. 4, No. 6, December 2012

it is performing some work at the shelter. If the total time for waiting at the shelter elapses then it moves on to the next shelter in its list i.e. it moves to the next unvisited shelter whose reference (name \& location) is present with it. It also keeps a check on this shelter's entry in its own table, so that it can pay a visit once again in the near future. But before leaving the shelter, it ensures to exchange any new information (present with it) with the shelter.

- If the flag is found false, then no one is working for this shelter and hence it gets an opportunity to work for this shelter. It then immediately makes the flag true and checks the status of the resource present at this shelter. If it is greater than equal to 0 then no need of going to the CS because there is no demand of resources. Instead leave this shelter and go to the next shelter in its list.

- Again if the resources are found negative, then it immediately goes to the CS to fetch resource for it, whereby the CS gives it a task to perform.

- A volunteer does not keep the record of the resources of previous entries of a shelter. It only keeps the status of the resources of the shelter that it is involved presently. A volunteer at a time associates with only one shelter. When a volunteer is involved with a shelter, at that moment only that volunteer can perform the tasks related to the shelter. No other volunteer can interfere to take charge of the tasks; but surely it can exchange information. A shelter similar to a volunteer is also associated to a single volunteer for resource updating at a time.

- When a volunteer comes to deliver negative resource information from a shelter to the CS, the CS first updates any new piece of information from the volunteer and then takes the recent resource status (negative) of the shelter it is coming from.

- After updating its own list of resource status of the shelters, it then runs an algorithm which finds out a nearby shelter (to the concerned shelter) having excess resource. If there is no such shelter with excess resource within the vicinity of the concerned shelter, then it gives out resources from itself and hence directs the volunteer with resources directly to the shelter in demand.

\subsection{Assumptions for simulation}

- There is a common synchronised watch available to all volunteers.

- The consumption of resources is instantaneous i.e. the resources are consumed immediately when they are being transferred to a shelter.

- The shelters are assumed to be formed before the central station can take notice of them and they have to be discovered by the volunteers.

- Volunteers carrying PDA / Smart-phone, supporting query / exchange information autonomously

\subsection{Simulation results}

- Figure 8 shows the individual resource status of the shelters in the affected area w.r.t. time-ticks. 


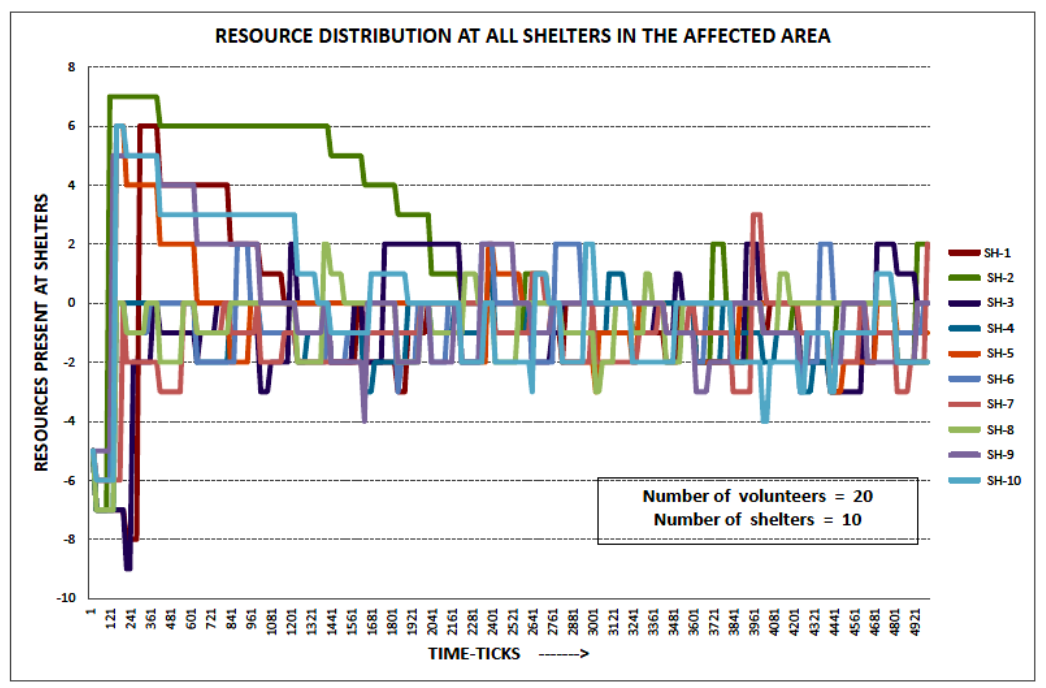

Figure 8. Resource distribution at all shelters in the affected area

- This graph is a direct consequence of the simulation with number of volunteers and shelters set at 20 and 10 respectively.

- The shelters possess self-decrement of resources which means there is a demand for resources at the shelters. The central station is assumed to be situated at an approximate distance of 3 kilometres from the affected area.

- It is deduced from the graph that initially there is a huge demand and it increases with the time-ticks till 250 to 300 ticks. Before this time the volunteers were busy discovering the unknown shelters that were formed previously in the affected area.

- The transferring of resources to a shelter is reflected in the graph when the net resource at a shelter increases from negative to 0 or to the double of the demand.

- The most interesting part is that as time increases, the demands of the shelters are replenished quickly as and when noticed by the volunteers. The graph also shows that majority of the shelters gets their demands fulfilled before their resource status reaches 3 . Most are replenished quickly at -1 and -2 before their demand increases further.

- From this graph we conclude that with the increase of demand for resources at shelters, the volunteers deliver the information to the central station, which in turn looks after the fact that no shelter starves for resources for a long time (apart from the time needed to fetch and transfer the resources). This is seen in the later phase of the graph where the initial fluctuating pattern is brought down to much more linear pattern, where the resources at any shelter is neither too large (in excess) nor is devoid of it for a long period of time.

- Figure 9 represents a screenshot of one of the simulation in REPAST taken at time tick $=250$. The number of volunteers working in the field and the number of shelters present are set to 20 and 10 respectively. The central station is situated at an approximate distance of 3 kilometres from the affected area. 
International Journal of Wireless \& Mobile Networks (IJWMN) Vol. 4, No. 6, December 2012

- In figure 9 we see that the volunteers (represented by small blue dots) moving towards and away from the Check-In-Area. Some volunteers had changed their color to red, which signifies that they are moving out from the Check-In-Area carrying resources for their respective shelters. The agents which oscillates between the Check-In-Area and the Fixed Tower (represented by small black dots), belongs to the Check-In-Area and are seen to move towards the Fixed Tower. They are transferring any new static information present at the Check-In-Area to the Fixed Tower.

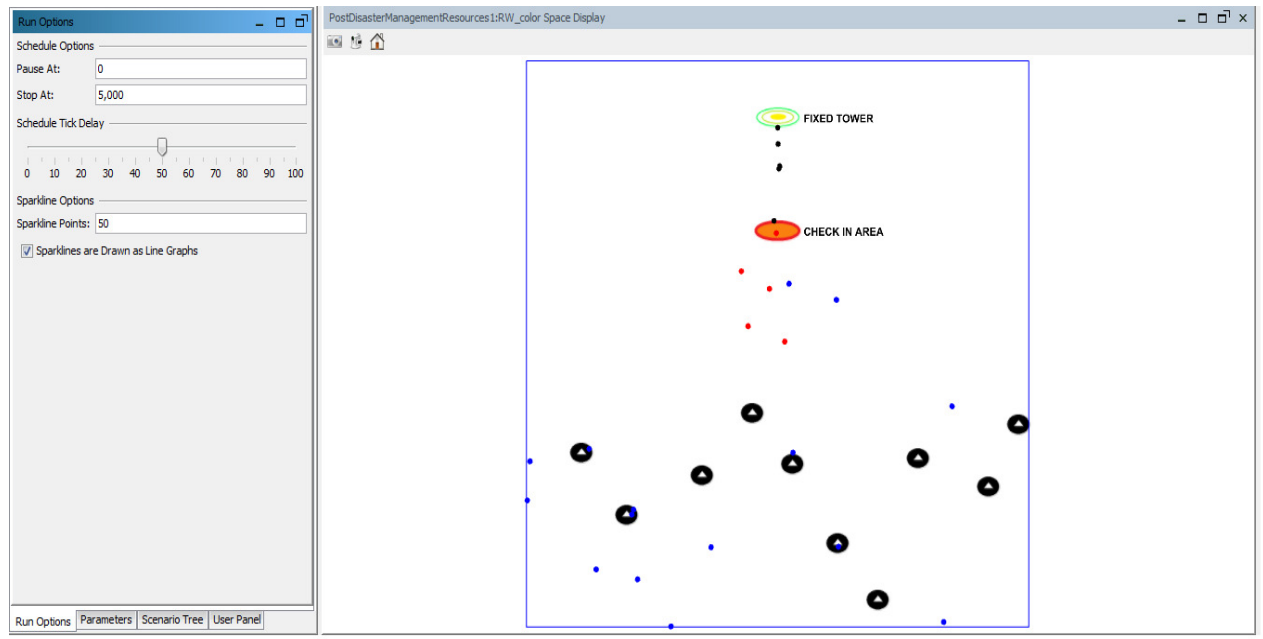

Figure 9. Screenshot of a simulation taken at time tick $=250$. The volunteer and shelter counts at the field is set to 20 and 10 respectively. The boundary specifies our area of interest and measures 10 kilometres x 10 kilometres.

- Next we have figure 10 which shows the perception of resources by the central station at the affected area after it supplied the resources vs. the actual resources present at the area.

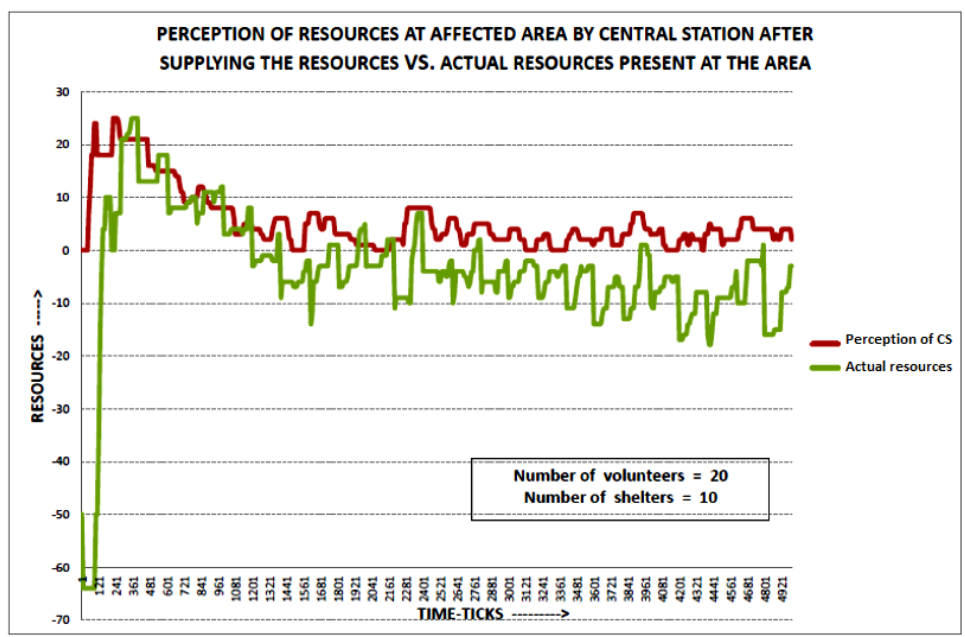

Figure 10. Perception of resources at affected area by central station after supplying the resources vs. Actual resources present at the area 
International Journal of Wireless \& Mobile Networks (IJWMN) Vol. 4, No. 6, December 2012

- The number of volunteers working in the affected area and the number of shelters are 20 and 10 respectively. The distance between the central station and the affected area is moderate. This graph refers to the same simulation with the same conditions as graph of figure 8.

- Initially the central station assumes that the sum total of all resources at shelters (if any) is 0 , before they are discovered. As the volunteers discover the shelters and notify the central station of the demands, the Central Station updates its own list accordingly.

- It is to be remembered that this graph is a result of post supplying the resources by the central station. From this graph (the red line) it is seen that the perception of resources is always positive and hence understood that the central station tries its best to supply and distribute resources throughout the affected area in an effective way by applying its algorithm on its list.

- However, the other graph (the green line) showing the actual resources in the affected area depicts a deviation from the central station's viewpoint. But this graph almost resembles the perception graph. This is due to the delay introduced in overcoming the distance of separation between the shelters and the central station. This similarity of pattern between both the graphs concludes that even if the central station is placed at a distance of 3 kilometres from the affected area, the plan is working out as planned by the central station.

- Figure 11 represents the variations of the sum total of all resources present in the affected area w.r.t. time-ticks by varying the volunteer count.

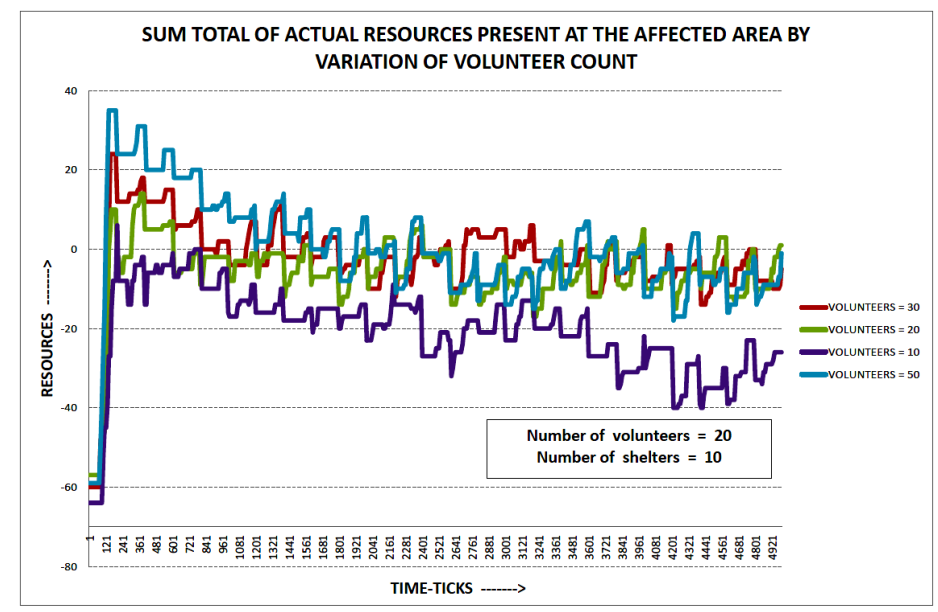

Figure 11. Sum total of actual resources present at the affected area by variation of volunteer count

- The graphs are plotted with volunteer counts of 10,20, 30 and 50 respectively. The shelter count remains constant for all the simulation and equals 10 .

- The simulations include the self-decrement of resources at shelters and the central station being placed at a distance of 3 kilometres from the affected area. 
International Journal of Wireless \& Mobile Networks (IJWMN) Vol. 4, No. 6, December 2012

- From the graph with volunteer count $=10$, we see that due to less number of volunteers, the volunteers do not get the opportunity of visiting the other shelters more frequently and are either busy searching for new shelters or is busy in a task. With less number of volunteers the shelters get starved.

- On the other hand we notice that the total resource distribution of the graphs with volunteers $=20,30$ and 50 falls within the same range i.e. the volunteer count values yields almost the same result in the long run.

- This justifies the fact that at some point, even if the volunteer's counts are increased, the net resource distribution at the affected area remains same. As depicted from the graphs, the optimum volunteer count in the affected area can be assumed to be 20 . Even if volunteers are increased beyond this value, the net distribution of resources will not be affected much.

- Next we have figure 12 which shows the convergence of static information (i.e. the number of volunteers and the number of shelters present) throughout the affected area w.r.t. time-ticks. These graphs are plotted to study the variation of the percolation of this information through every shelter and volunteer in the area.

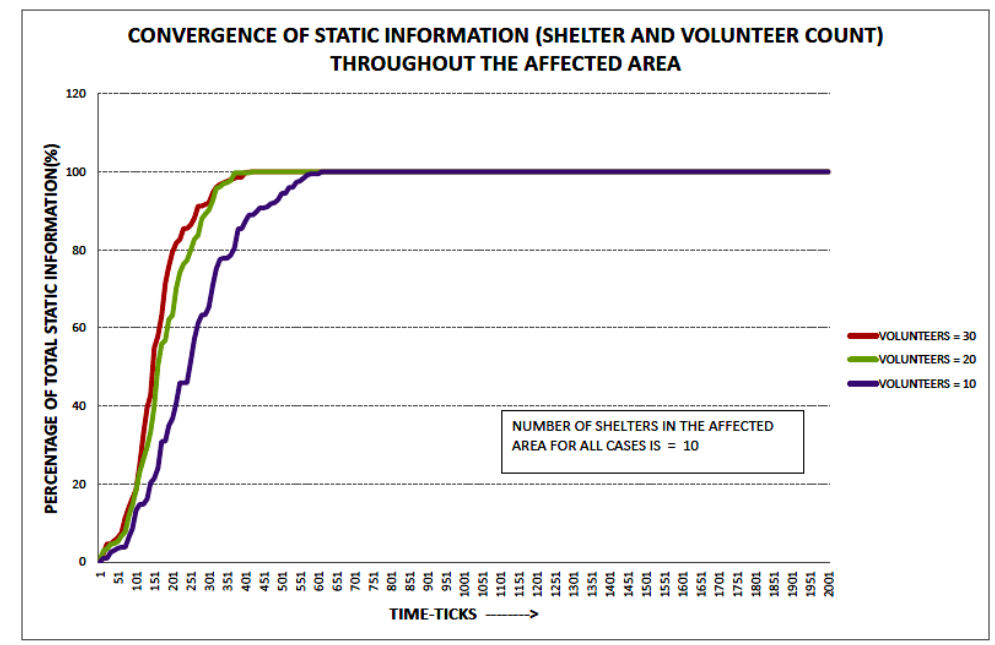

Figure 12. Convergence of static information (shelter and volunteer count) throughout the affected area

- The number of shelters is kept constant at 10 and the volunteer counts are varied by values 10, 20 and 30 . The $\mathrm{Y}$-axis represents the percentage of convergence of the total static information. For this we have taken the percentage of the information every volunteer and shelter carries individually and then take an average to find the aggregate percentage. The resulting graphs are inverted exponential graphs and the study reveals that as the volunteer counts increases, the time required to percolate the information throughout decreases to a certain point beyond which increasing the volunteer count yields the same result, i.e. the $100 \%$ convergence point coincides for values beyond 20 . 


\section{Conclusions}

In this paper, we have proposed an effective scheme of providing a coordinated post disaster relief operation. Assuming the absence of a network infrastructure, we tried to incorporate the notion of opportunistic network by using peer-to-peer communication between relief workers. We investigated the schema by varying key parameters that affects the performance of the system. The simulation results of the system shows that even if the entire coordinated operations are centralized and is monitored far away from the affected area, the operations yields positive results in the overall resource present at the affected area. We have also shown that the perception of resource pattern bears a similar trend to the actual resource pattern at the affected area proving the fact that information is percolated and perceived properly. Furthermore, we have found out the optimal relief worker count and proved that this optimal value has a direct impact on the convergence of static information throughout the affected area as information is dispersed quickly. We have also shown that resources are effectively distributed as and when demand arises at the shelters. The findings of this paper suggests that even in the absence of a network infrastructure, using peer-to-peer communication and message passing can eventually form an intermittent opportunistic network which performs effectively even if the key parameters are varied widely. This study thus reveals the applicability of the scheme in PDAs and smart-phones which can be used to form peer-to-peer wireless network during relief operations.

\section{REFERENCES}

[1] H. Luo, R. Kravets, T. Abdelzaher, The-Day-After Networks: A First-Response Edge-Network Architecture for Disaster Relief, NSF NeTS FIND Initiative, 2006-2010. $<$ http://www.nets-find.net/Funded/DayAfterNet.php>.

[2] Marco Conti, Mohan Kumar: Opportunities in Opportunistic Computing. IEEE Computer 43(1): 42-50 (2010)

[3] Conti, M.; Giordano, S.; May, M.; Passarella, A.; From opportunistic networks to opportunistic computing, IEEE Communications Magazine, IEEE, Volume: 48 Issue:9, Sept. 2010

[4] [Erik Auf der Heide, 1989]. Disaster Response - Principles of Preparation and Coordination, Publisher: Elsevier-Medical, 1989, p. 303

[5] [Gorp, 2008]. Annemijn van Gorp et al, "VSAT Deployment for Post-Disaster Relief and Development: Opportunities and Constraints for Inter-Organizational Coordination among International NGOs", 17th Biennial Conference, 2008.

[6] [Silvia, 2003], Suárez Silvia, López Beatriz, De la Rosa Josep Lluis. "MCD Method for resource distribution in a large-scale disaster". In: "X Conferencia de la Asociación Española para la Inteligencia Artificial" CAEPIA 2003, volumen II, pág. 261-264, San Sebastián, Spain, November $11-14$ of 2003.

[7] [Sonia, 2009]. Sonia Majid, Kazi Ahmed, "Cluster-based Communications System for Immediate Post-disaster Scenario”, Journal of Communications, Vol. 4, No. 5, June 2009.

[8] [Iannella, 2007]. R. Iannella and K. Henricksen, "Managing Information in the Disaster Coordination Centre: lessons and opportunities", in ISCRAM 2007 Conference, Delft, The Netherlands, May 2007.

[9] [Marrella, 2011]. Andrea Marrella et al, "Collaboration On-the-field: Suggestions and Beyond", in ISCRAM 2011 Conference, Lisbon, Portugal, May 2011.

[10] [de Leoni, 2007]. de Leoni et al., "Emergency Management: from User Requirements to a Flexible P2P Architecture", In Proceedings of ISCRAM 2007, 2007. 
International Journal of Wireless \& Mobile Networks (IJWMN) Vol. 4, No. 6, December 2012

[11] Howe, T.R., N.T. Collier, M.J. North, M.T. Parker, and J.R. Vos, "Containing Agents: Contexts, Projections, and Agents," Proceedings of the Agent 2006 Conference on Social Agents: Results and Prospects, Argonne National Laboratory, Argonne, IL USA (September 2006).

[12] North, M.J., P. Sydelko, J.R. Vos, T.R. Howe, and N.T. Collier, "Legacy Model Integration with Repast Simphony," Proceedings of the Agent 2006 Conference on Social Agents: Results and Prospects, Argonne National Laboratory, Argonne, IL USA (September 2006).

[13] Parker, M.T., T.R. Howe, M.J. North, N.T. Collier, and J.R. Vos, "Agent-Based Meta-Models," Proceedings of the Agent 2006 Conference on Social Agents: Results and Prospects, Argonne National Laboratory, Argonne, IL USA (September 2006).

[14] Tatara, E., M.J. North, T.R. Howe, N.T. Collier, and J.R. Vos, "An Introduction to Repast Modelling by using a Simple Predator-Prey Example," Proceedings of the Agent 2006 Conference on Social Agents: Results and Prospects, Argonne National Laboratory, Argonne, IL USA (September 2006).

[15] A Survey of Opportunistic Networks, Chung-Ming Huang, Kun-chan Lan22nd International Conference on Advanced Information Networking and Applications - Workshops, 978-0-76953096-3/08 \$25.00 @ 2008 IEEE, DOI 10.1109/WAINA.2008.292, 1672

\begin{abstract}
Authors
Somprakash Bandyopadhyay is a Professor in Management Information Systems Group of Indian Institute of Management, Calcutta. He is also in the Board of Directors of Pervcom Consulting. He is a fellow of the Alexander von Humboldt Foundation, Germany and fellow of the Japan Trust International Foundation. He has around 25 years of experience in teaching, research and software development in several organizations of international repute, including PricewaterhouseCoopers Ltd., Advanced Telecommunications Research Institute, Japan, Indian Institute of Technology Kharagpur, Indian Institute of Technology Bombay, German Research Centre for Artificial Intelligence, Germany, Tata Institute of Fundamental Research, Bombay and Jadavpur University, Calcutta. He has initiated Ad Hoc Network Research Group in 1998 to provide an environment for research and application development on wireless and mobile communication systems.
\end{abstract}

Sagar Bose is a Senior Product Manager of PervCom Consulting Pvt. Ltd., Calcutta, India. He is a B.Tech in Electronics and Communication Engineering and MBA in Marketing Management. He has around seven years of experience in teaching, research, software \& hardware development in several organizations of international repute, including Indian Institute of Management Calcutta, India. He has functional experience of Active RFID, Passive RFID, Wireless Sensor Network, Embedded Systems, Customer Relationship Management, Product Management and Marketing Management. He has been actively involved in the designing, development, testing and debugging of applications in various projects.

Debanjan DasDeb has just received his B.Tech in Computer Science and Engineering from B.P.Poddar Institute of Management and Technology. He has an interest in representing complex computing systems with agent-based models. His interest also lies in artificial intelligence, robotics and embedded systems. He has functional experience in the field of embedded systems.

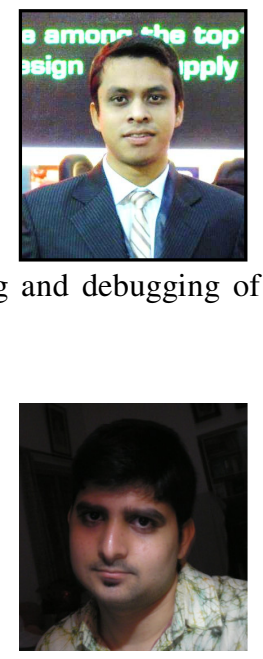

